\title{
Laser-induced periodic annular surface structures on fused silica surface
}

\author{
Yi Liu ${ }^{1, *}$, Yohann Brelet ${ }^{1}$, Zhanbing $\mathrm{He}^{2}$, Linwei $\mathrm{Yu}^{3}$, Benjamin Forestier ${ }^{1}$, Yongkai Deng ${ }^{4}$, Hongbing Jiang ${ }^{4}$, \\ and Aurélien Houard ${ }^{1, *}$
}

\begin{abstract}
${ }^{l}$ Laboratoire d'Optique Appliquée, ENSTA/CNRS/Ecole Polytechnique, 828, Boulevard des Maréchaux, Palaiseau, F-91762, France
${ }^{2}$ Electron Microscopy for Materials Research (EMAT), University of Antwerp, Groenenborgerlaan 171, Antwerp B-2020, Belgium

${ }^{3}$ Laboratoire de Physique des Interfaces et des Couches Minces, Ecole Polytechnique, CNRS, Palaiseau, F-91128, France

${ }^{4}$ Department of Physics and State Key Laboratory for Mesoscopic Physics, Peking University, Beijing 100871, China *yi.liu@ensta-paristech.fr; aurelien.houard@ensta-paristech.fr
\end{abstract}

\begin{abstract}
We report on the formation of laser-induced periodic annular surface structures (LIPASS) on fused silica irradiated with multiple femtosecond laser pulses. This novel morphology emerges after the disappearance of the conventional laser induced periodic surface structures (LIPSS), under successive laser pulse irradiation. It is independent of the laser polarization and universally observed for different focusing geometries. We interpret its formation in terms of the interference between the reflected laser field on the surface of the damage crater and the incident laser pulse.
\end{abstract}

Lasers-induced periodic surface structures (LIPSS), also referred to as ripples, were discovered 40 years ago on Germanium upon irradiation with linearly polarized laser pulses [1]. After that, LIPSS was observed on almost every solid, including semiconductors, metals, and dielectrics under the irradiation of pulsed or continuous laser field at various wavelengths [2-6]. Therefore, it is widely deemed as a universal phenomenon $[4,5]$. The morphology of these structures depends on the laser polarization, incident angle, and the nature of the materials. With linearly polarized laser pulses incident normally on the sample surface, regular ripples with period $(\Lambda)$ close to the laser wavelength $(\lambda)$ are usually observed. The ripples can orientate parallel or orthogonal to the polarization direction of the laser field, depending on the material, which are referred to as c-type and s-type respectively $[4,5]$. On the other hand, the damaged surface exhibits speckled structure without any predominant orientation under the irradiation of circularly polarized laser [5]. The commonly accepted underlying mechanism for LIPSS is the interference of the incident laser field with some form of surface scattered electromagnetic wave $[4,5]$.

The study of LIPSS has been revived around the year 2000 when periodic structures with periods much shorter than $\lambda / 2$ were observed on the surface or in the bulk of dielectrics following irradiation with several thousands of femtosecond laser pulses [7-9]. Later, it was observed on the surface or in the volume of metals, semiconductors, and various dielectrics for moderate pulse numbers [10-18]. The observed periods reached values in the range $\lambda / 5-$ $\lambda / 10[11,14,18]$. Conventionally, these shorter period ripples are named as High Spatial Frequency LIPSS (HSFL) and ripples with period close to $\lambda$ as Low Spatial Frequency LIPSS (LSFL) $[11,14-18]$. The interpretation of the HSFL is controversial. Several interpretations have been proposed, such as self-organization [7], interference between laser field and plasma wave or surface plasmons [9, 13, 15], local field enhancement due to the nanoplasma [10], and second harmonic generation (SHG) [14].

In this letter, we report on a novel type of low spatial frequency surface structure, namely Laser-Induced Periodic Annular Surface Structures (LIPASS). We found that the traditional LIPSS disappears gradually under successive laser pulse irradiation when the crater deepens. 
Instead, the LIPASS forms in the center of the damage crater. Different from the LIPSS, the LIPASS is independent of laser polarization. We attribute the formation of LIPASS to the interference between the incident laser field and that reflected on the crater walls. Our interpretation is supported by the fact that the LIPASS transforms to nearly parallel ripples structure when an elliptic damage crater is formed, which is realized by introducing astigmatism in the laser beam.

In our experiments, femtosecond laser pulses (50 fs, $800 \mathrm{~nm}$ ) from a chirped pulse amplification (CPA) system are focused by different convex lenses in ambient air. The fused silica sample is mounted on a 3-dimensional translation stage, which allows a movement to a fresh site after each series of laser irradiation. The polarization of the pulses can be changed with a $\lambda / 4$ waveplate before the focusing lens. After laser irradiation, the sample was cleaned in a supersonic methanol bath and coated with gold to increase its surface conductivity. The sample was then examined by a scanning electron microscope (SEM, JEOL 650).

Figure 1 presents the morphology of the damage as a function of laser shot numbers $N$. A lens with focal distance $f=75 \mathrm{~mm}$ was used and the beam waist $\left(1 / \mathrm{e}^{2}\right)$ in the focal plane was measured to be $13.5 \mu \mathrm{m}$. The incident pulse energy was $28 \mu \mathrm{J}$, which corresponds to a peak laser fluence of $F=9.8 \mathrm{~J} / \mathrm{cm}^{2}$ at the sample surface. After 10 laser shots, well aligned surface ripples with period around $0.7 \mu \mathrm{m}$ were observed (Fig. 1(a)). With increase of laser shots number $N$, the LIPSS on the periphery of the ablated site becomes less significant and finally dissolves. This peripheral area is instead covered by sub-micron rods and particles without obvious spatial periodicity (Fig. 1(b) and (c)). Progressive decrease of the LIPSS area towards the center was observed with further increase of the laser shots number $N$. Finally, the LIPSS totally vanish after $N>50$ (Fig. 1(d)). Interestingly, after $N=100$ a dip with diameter of $\sim 2$ $\mu \mathrm{m}$ is observed in the center of the damage, which is surrounded by several annular periodic fringes (Fig. 1(e)). The period of these annular fringes is about $0.6-0.7 \mu \mathrm{m}$, with a slight decrease from the most inner one to the outside. In view of this spatial characteristic, we name it Laser-induced Periodic Annular Surface Structure (LIPASS). The LIPASS remains quite stable upon further laser shots, as evidenced by the morphology with $N=550$.

We first examined the influence of incident laser energy on the formation of LIPASS. In Fig. 2 (a) and (b), the morphology of the laser damages for pulses of $19 \mu \mathrm{J}$ and $9 \mu \mathrm{J}(F=6.6$ and $3.1 \mathrm{~J} / \mathrm{cm}^{2}$ ) are presented respectively. Similar LIPASS pattern are observed. We next examined the influence of laser polarization. With elliptically and circularly polarized laser pulses, we observed almost identical LIPASS in experiments. The result for circular polarization is presented in Fig. 2 (c). The independence of the LIPASS formation on laser polarization is totally different from the traditional LIPSS formation, which is sensitive to laser polarization.

To check the LIPSS formation with other focusing geometries, we changed the focal lens from $f=75 \mathrm{~mm}$ to $200 \mathrm{~mm}, 500 \mathrm{~mm}$ and $1000 \mathrm{~mm}$. For all these three focusing geometries, similar LIPASS was observed for proper incident laser energy. For example, the result for $f=$ $1000 \mathrm{~mm}$ is presented in Fig. 2 (d). The LIPASS in this case was found to be less symmetrical. This can be due to the minor astigmatism inherent to our laser system, whose influence is more significant for relatively loose focusing. We will come back to this point at the end of the manuscript.

To get insight into the underlying mechanism for the formation of LIPASS, we measured the profile of the crater with a profilometer (Dektek 150). The result corresponding to Fig. 1(f) is 
presented in Fig. 3. The crater exhibits a cone shape and its depth reaches $\sim 10 \mu \mathrm{m}$ after 550 laser shots.

The independence of the LIPASS formation on the laser polarization excludes the traditional mechanism of LIPSS $[4,5]$. With the above observation, we attributed it to the interference of the reflected laser field on the crater surface with the incident laser field. It has been noted that during laser ablation the reflectivity of the surface can reach $\sim 30 \%$ due to the presence of dense electronic plasma [19]. We presented a schematics model in Fig. 4 (a), where a cross section of the conical crater is represented by a triangle with an apex angle of $\phi$. In the o-e-c region(shaded red in Fig 4(a)), the reflected laser field from the upper side of the crater surface with wave vector $\vec{k}_{2}$ interferes with the incident laser field with wave vector $\vec{k}_{0}$, leading to periodic laser intensity modulation perpendicular to $\vec{k}_{0}-\vec{k}_{2}$. The spatial period of this interference is

$$
\Lambda_{1}=\lambda /(2 \sin (\theta / 2))
$$

where $\theta=\phi$ is the crossing angle between the two laser fields. After some geometrical considerations, it was found that the lateral period $\Lambda_{2}$ of the surface fringe written by the above laser intensity modulation is

$$
\Lambda_{2}=\Lambda_{1} / \sin ((\theta+\phi) / 2)
$$

The periodicity observed in the SEM image is a projection of the surface fringe on the bottom plane of the cone and its period reads as:

$$
\Lambda_{3}=\Lambda_{2} \sin (\phi / 2)
$$

From Fig. 3, the crossing angle of the two laser fields is estimated to be $\sim 35^{\circ}-50^{\circ}$ in our experiment for $N=550$. The corresponding spatial period $\Lambda_{3}$ is then found to be $0.8-0.52 \mu \mathrm{m}$, which agrees well with our observations. In Fig. 4(b), we presented the calculated laser intensity distribution on the inner surface of the crater. A collimated Gaussian beam with beam waist $\omega_{0}=13.5 \mu \mathrm{m}$ was considered to illuminate the cone with a bottom surface diameter $D=16 \mu \mathrm{m}$. The apex angle was taken to be $\phi=40^{\circ}$ and a surface reflectivity of $30 \%$ was considered. Intensity modulation with a period of $0.62 \mu \mathrm{m}$ in the radial direction is observed, which is in good agreement with the result presented in Fig. 1(f). It is also worth noticing that a strong intensity peak locates at the very center, which explains the central hole of the morphology presented in Fig. 1 (e)-(f) and Fig. 2(a)-(d).

Based on the above interpretation, one would expect that the form of the LIPASS should change if the crater is not symmetrical. To verify this, we intentionally introduced astigmatism on the incident laser beam by tilting the focusing lens with an angle $\varphi \sim 5^{\circ}$. It is well known that in the presence of astigmatism the focus regime is featured as two elliptical intensity profiles before and after the focus, while the focus remains round with an increased waist size. We performed damage experiment $\sim 1.5 \mathrm{~cm}$ after the focus, where the beam is elongated in the vertical direction. The SEM images of the corresponding damage and a zoom of the central area are presented in Fig. 5. The damage shapes now transforms into a vertically orientated ellipse. More important, several nearly parallel ripples appear on the damage center, orientated in the direction of the long axis of the damage ellipse. It can be easily explained by the fact that the crater now takes a biprism shape and the corresponding interference fringe naturally orientates along the long axis of the crater.

In summary, we observed LIPASS formation on the center of the laser ablation crater after the disappearance of the traditional LIPSS under successive laser shots. The LIPASS is found to be independent of the polarization of the laser pulses. We proposed the interference of the 
incident laser field and the reflected laser field on the crater surface is responsible for this new morphology. We also demonstrated that LIPASS can be turn into nearly parallel ripples when an elliptic damage is produced in presence of focusing astigmatism, which further confirms our interpretation.

Acknowledgement:

Y. L. is grateful to Jörn Bonse for stimulating discussion. The project has been partially funded by Contract No. ANR-2010-JCJC-0401-01.

Reference:

[1] M. Birnbaum, J. Appl. Phys. 36, 3688 (1965).

[2] D. C. Emmony, R. P. Howson, and L. J. Willis, Appl. Phys. Lett. 23, 598 (1973).

[3] P. M. Fauchet and A. E. Siegman, Appl. Phys. Lett. 40, 824 (1982).

[4] H. M. Van Driel, J. E. Sipe, and J. F. Young, Phys. Rev. Lett. 49, 1955 (1982).

[5] J. F. Young, J. S. Preston, H. M. van Driel, and J. E. Sipe, Phys. Rev. B 27, 1155 (1983).

[6] Y. Liu, Y. Brelet, Z. He, L.Yu, S. Mitryukovskiy, A.Houard, B. Forestier, A. Couairon, and A. Mysyrowicz, Phys. Rev. Lett. 110, 097601(2013).

[7] J. Reif, F. Costache, M. H. Genyk, S. V. Pandelov, Appl. Surf. Sci. 197, 891 (2002).

[8] Q. Wu, Y. Ma, R. Fang, Y. Liao, Q. Yu, X. Chen, and K. Wang, Appl. Phys. Lett. 82, 1703(2003).

[9] Y. Shimotsuma, P. G. Kazansky,J. Qiu, K. Hirao, Phys. Rev. Lett. 91, 247405 (2003).

[10] V. R. Bhardwaj, E. Simova, P. P. Rajeev, C. Hnatovsky, R. S. Taylor, D. M. Rayner, and P. B. Corkum, Phys. Rev. Lett. 96, 057404 (2006).

[11] M. Shen, J. E. Carey, C. H. Crouch, M. Kandyla, H. A. Stone, and E. Mazur, Nano Lett. 8, 2087 (2008).

[12] T. Y. Hwang and C. Guo, Opt. Lett. 36, 2575 (2011).

[13] M. Huang, F. Zhao, Y. Cheng, N. Xu, and Z. Xu, ACS Nano, 3, 4062 (2009).

[14] T. Q. Jia, H. X. Chen, M. Huang, F. L. Zhao, J. R. Qiu, R. X. Li, Z. Z. Xu, X. K. He, J. Zhang, and H. Kurodu, Phys. Rev. B 72, 125429 (2005).

[15] L. Xue, J. J. Yang, Y. Yang, Y. S. Wang, X. N. Zhu, Appl. Phys. A 109, 357(2012).

[16] H. L. Ma, Y. Guo, M. J. Zhong, and R. X. Li, Appl. Phys. A 89, 707(2007).

[17] F. Liang, R. Vallée, and S. L. Chin, Opt. Express 20, 4389 (2012).

[18] S. Hohm, A. Rosenfeld, J. Kruger and J. Bonse, J. Appl. Phys. 112, 014901(2012).

[19] H. O. Jeschke, M. E. Garcia, and K. H. Bennemann, Phys. Rev. B 60, R3701 (1999). 

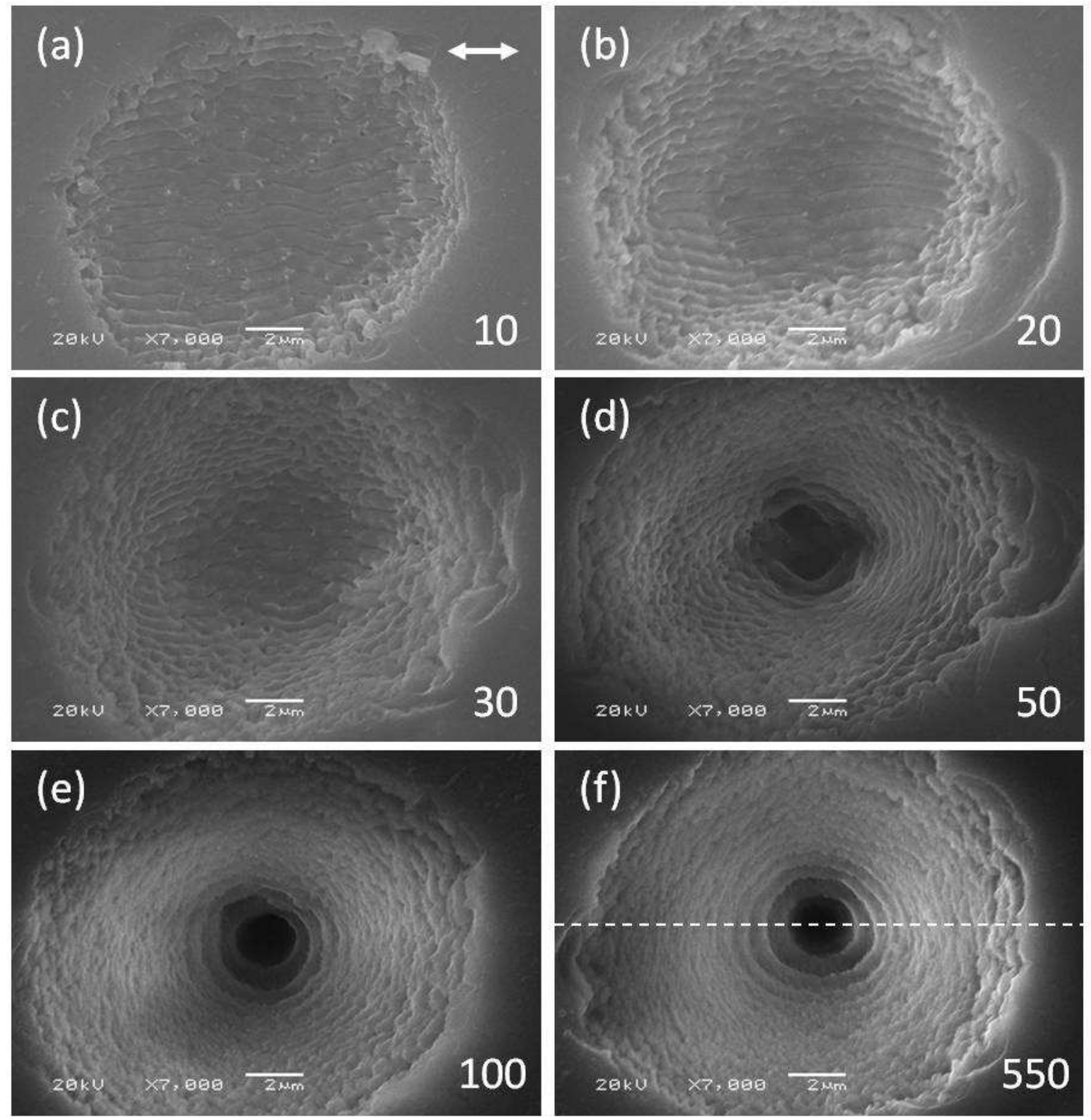

Fig. 1. Evolution of the damage morphology as a function of the laser shots number. The numbers on each panel indicate the laser shots numbers. In (a), the arrow represents the direction of laser polarization. In (f), the horizontal dashed line presents the position of the cross section profile measurement presented in Fig. 3. 

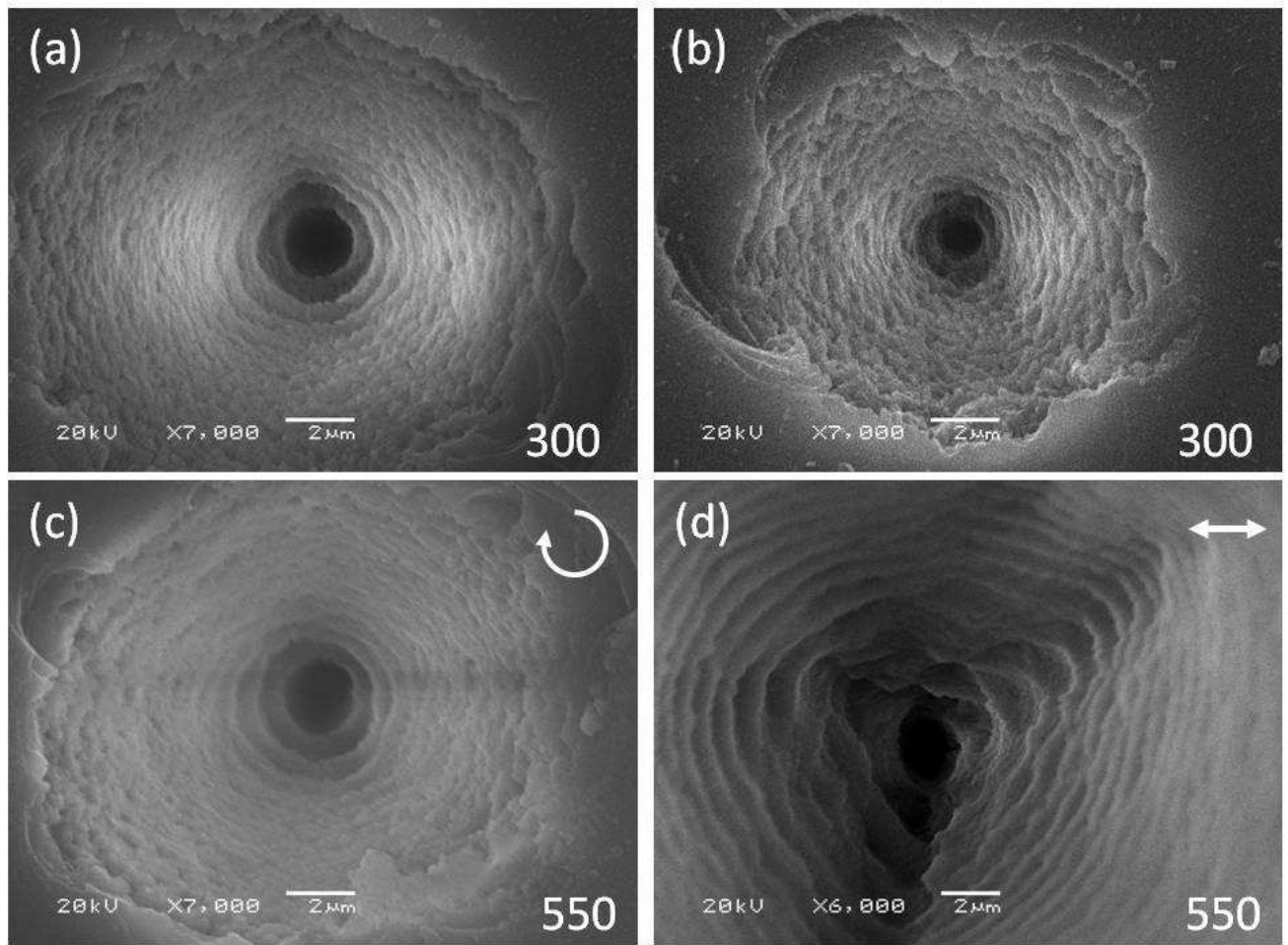

Fig. 2. The incident pulse energies are $19 \mu \mathrm{J}$ and $9 \mu \mathrm{J}$ for (a) and (b). The other experimental conditions are identical to those in Fig. 1. (c) The laser polarization is changed to circular. (d) The focal length is $f=1000 \mathrm{~mm}$ and the incident pulse energy is $300 \mu \mathrm{J}$. In each panel, the numbers indicate the laser shot number. In (c) and (d), the arrows present the polarization state of the laser pulses. 


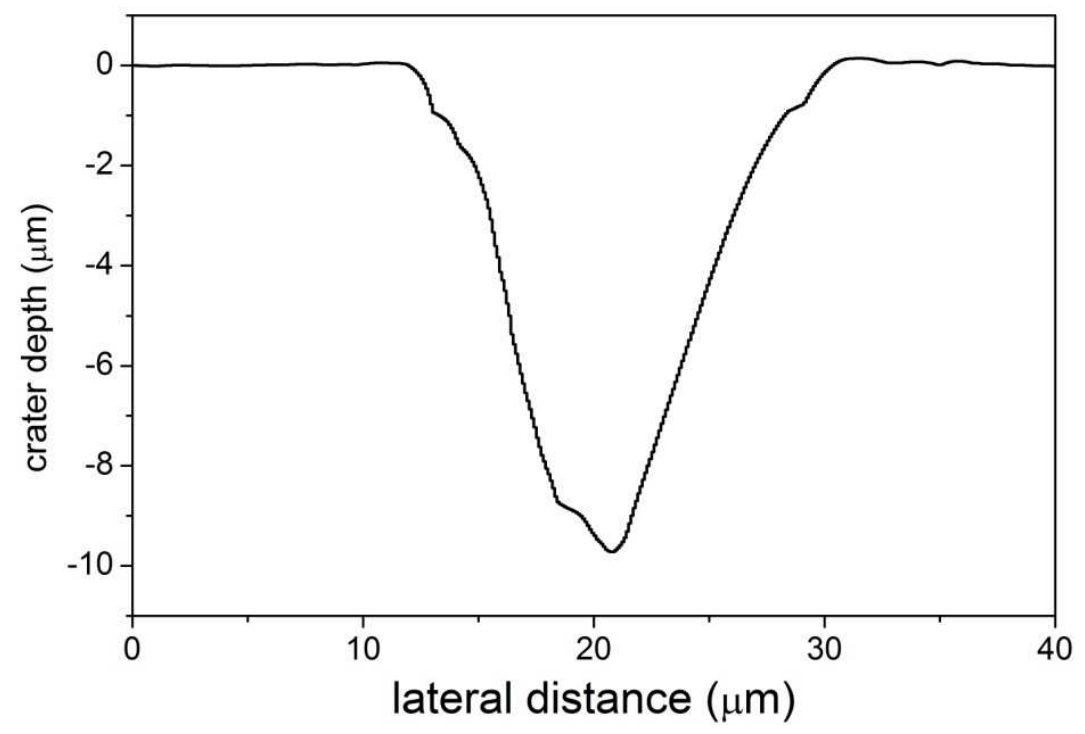

Fig. 3. Measured cross section profile of the crater presented in Fig. 1 (f). The position of the measurement is indicated by the dashed line in Fig. 1 (f). 

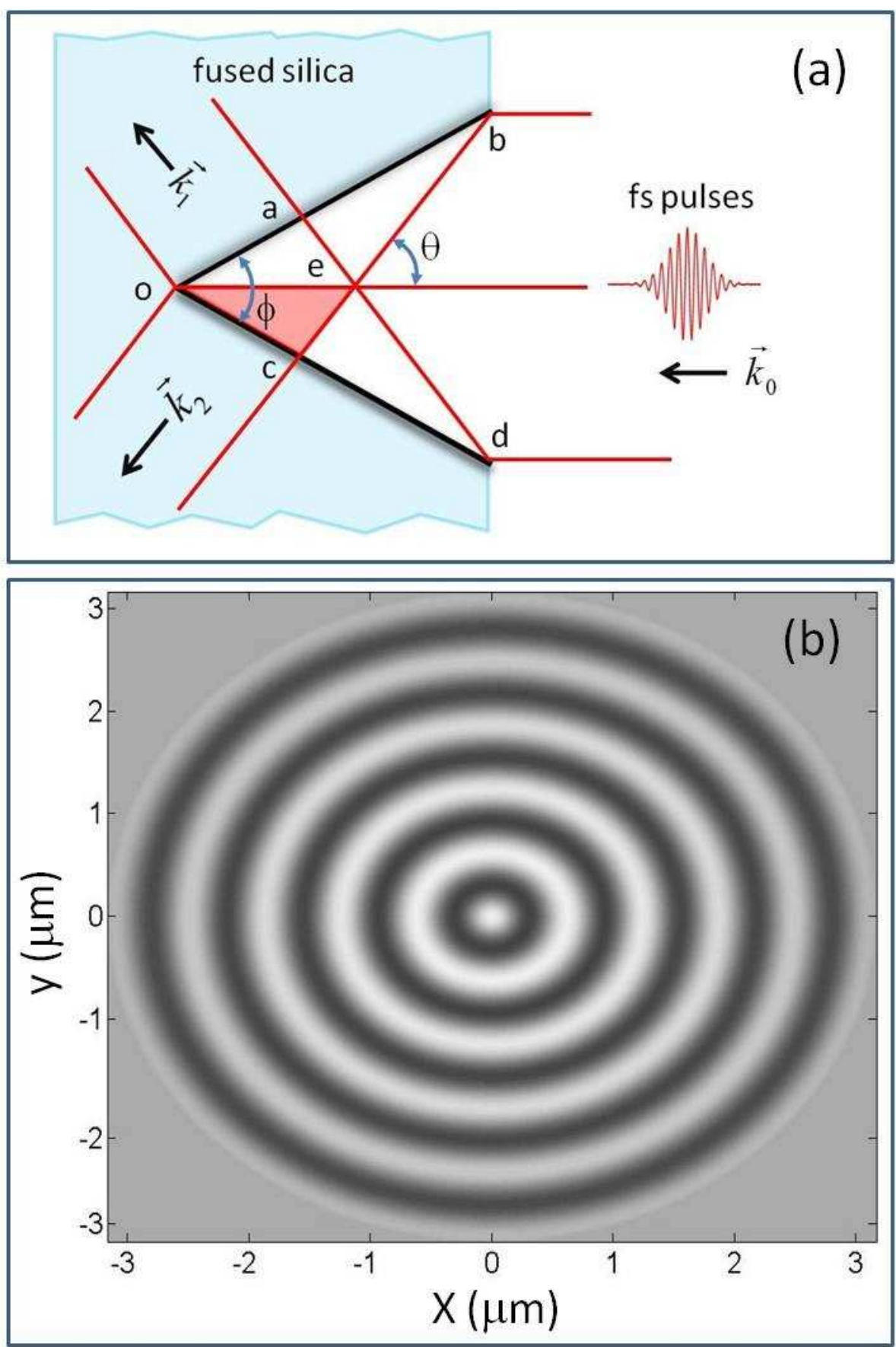

Fig. 4. (a) Schematic of the model. (b) Calculated intensity distribution of the interference laser field on the inner side surface of the damage crater. 

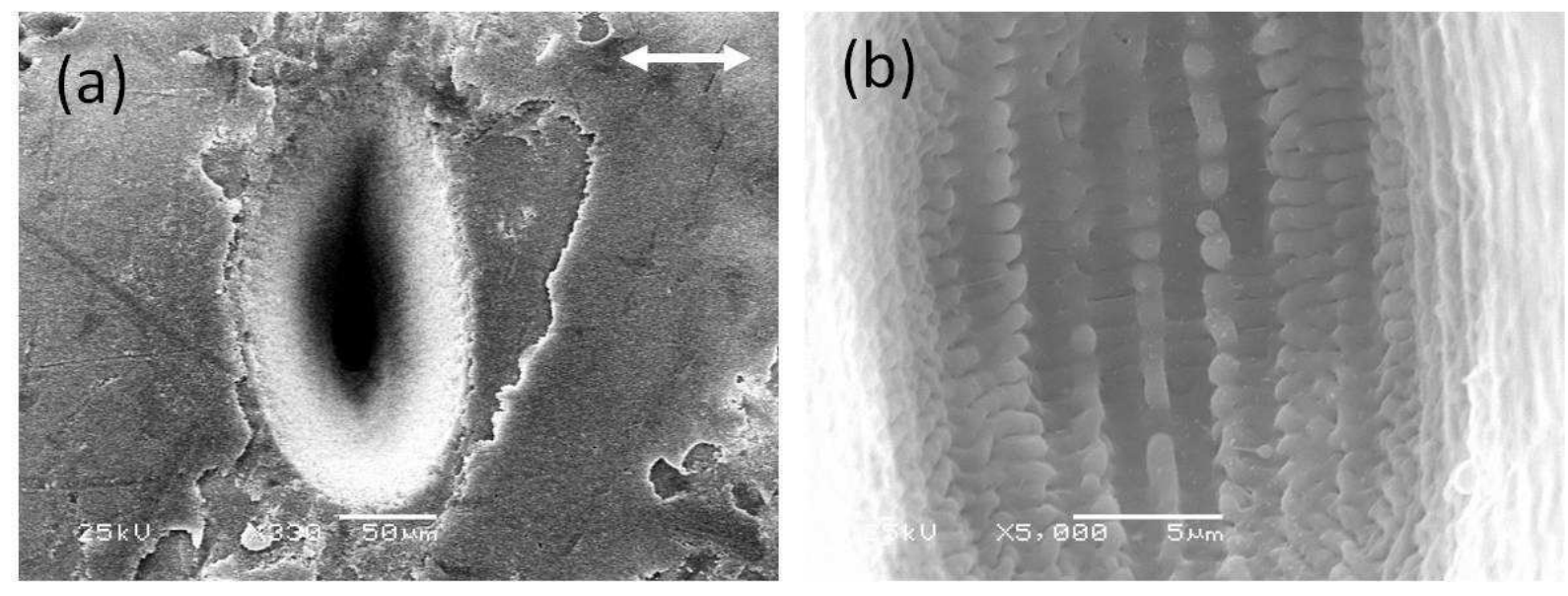

Fig. 5. The laser pulses are focused by $f=1000 \mathrm{~mm}$ lens with additional astigmatism. (a) Whole view of the damage crater. The arrow represents the polarization direction of the laser pulse. (b) Higher resolution SEM image of the central section of (a). Vertical fringe structure with period around 2-3 $\mu \mathrm{m}$ is clear, in addition to the horizontal LIPSS with period of $\Lambda \sim 0.7 \mu \mathrm{m}$. 